\title{
Space-air-ground integrated networks for future loT: Architecture, management, service and performance
}

\author{
Feng Lyu ${ }^{1} \cdot$ Wenchao Xu ${ }^{2} \cdot$ Quan Yuan $^{3} \cdot$ Katsuya Suto ${ }^{4}$ \\ Received: 22 April 2021 / Accepted: 22 April 2021 / Published online: 15 May 2021 \\ (C) The Author(s), under exclusive licence to Springer Science+Business Media, LLC, part of Springer Nature 2021
}

\section{Guest Editorial:}

With the emerging applications of ubiquitous Internet of Things (IoT), autonomous driving, and virtual reality (VR)/augmented reality (AR), future networks are expected to enable ultra-reliable and low-latency communications (URLLC), and support massive simultaneous access with extremely high data rates ( 10Gbps). Terrestrial networks, e.g., the well-deployed $4 \mathrm{G}$ or the emerging $5 \mathrm{G}$ cellular networks, are unable to fully satisfy the Quality of Services (QoS) due to their limitations, such as the inflexibility of fixed ground infrastructure, coverage holes, and rigid resource feeding manner, etc. Space networks (e.g., low Earth orbit (LEO) satellites) and aerial networks (e.g., unmanned aerial vehicles (UAVs)) can be leveraged to extend the scope of the terrestrial networks and constitute the space-air-ground integrated networks (SAGIN) ingeniously. Particularly, in SAGIN, in addition to the terrestrial networks, the satellites can provide ubiquitous wireless coverage cost-effectively, and the on-

This article is part of the Topical Collection: Special Issue on Space-AirGround Integrated Networks for Future IoT: Architecture, Management, Service and Performance

Guest Editors: Feng Lyu, Wenchao Xu, Quan Yuan, and Katsuya Suto

Feng Lyu

fenglyu@csu.edu.cn

Wenchao $\mathrm{Xu}$

wcxu@cihe.edu.hk

Quan Yuan

yuanquan@bupt.edu.cn

Katsuya Suto

k.suto@uec.ac.jp

1 Central South University, Changsha, China

2 Caritas Institute of Higher Education, Tseung Kwan O, Hong Kong

3 Beijing University of Posts and Telecommunications, Beijing, China

4 The University of Electro-Communications, Tokyo, Japan demand deployment of aerial networks can enhance the network flexibility and adaptability to various and dynamic environments, collectively ensuring the network QoS in supporting IoT services.

Despite the promising merits of SAGIN, technical and in-depth researches for SAGIN are urgently required. On the one hand, the heterogeneous network resources that have distinct features in terms of spectrum usage, access technology, transmission performance, and mobility characteristic, can render the operation and management of network intricate, which challenges the crucial construction of network architecture, resource management, and protocol design. On the other hand, to fully unleash the potential of SAGIN, the novel service provision, new computing-communication integrated paradigms, and performance modeling and analysis in SAGIN, need further investigation.

This special issue aims to bring together state-of-the-art research contributions that push forward the technological developments related to SAGIN enabled IoT, which will focus on the SAGIN network architecture design and implementation, resource management and orchestration, softwaredefined SAGIN, edge computing/ intelligence, IoT services, performance analysis and evaluation, and other promising technologies in SAGIN enabled IoT.

The response to our call for this special issue was significant, as we received in total 22 submissions from around the world. During the review process, each article was assigned to and reviewed by at least three experts in the field, with a rigorous multi-round review process. Thanks to the great support from the Editor-in-Chief, Prof. Xuemin (Sherman) Shen, and the dedicated work of numerous reviewers, we were able to accept 9 highquality articles covering various topics in SAGIN. In what follows, we will introduce these articles with highlighting their contributions.

In this first article "A Distributed Matching Game for Exploring Resource Allocation in Satellite Networks", Mi et al. proposed a novel one-to-one matching model under 
bilateral preferences in order to satisfy the QoS requirements with limited resources, and then devised the TaskOriented Gale-Shapley (T-O GS) algorithm and Adjacent Time Slot Matching (ATSM) algorithm to achieve the stable matching dynamically.

In the article "Cooperative Content offloading Scheme in Air-Ocean Integrated Networks", Zhou et al. proposed a Q-learning and game based unmanned aerial vehicles (UAVs) and unmanned surface vehicles (USVs) cooperation content offloading scheme in the air-ocean integrated networks (AOINs), which can significantly improve the use efficiency of UAV and increase the utility of USV compared with the traditional schemes.

In the article "Joint Association and Power Optimization for Multi-UAV Assisted Cooperative Transmission in Marine IoT Networks", Lyu et al. proposed a multiUAV assisted cooperative transmission to maximize the total throughput under the constrained of outage probability, transmit power and available channels, where they analyzed the achievable transmission reliability of the USV-OBS link, and the USV-UAV association and the transmit power control are jointly optimized to maximize the total transmission throughput for environment monitoring in marine IoT systems.

In the article "Downlink Aware Data Scheduling with Delay Guarantees in Resource-Limited LEO Satellite Networks", Wan et al. proposed a downlink aware data scheduling algorithm based on a decomposition strategy of rolling horizon mechanism, which can improve the network profit with guaranteeing the transmission delay.

In the article "A Double-layer Collaborative Apportionment Method for Personalized and Balanced Routing", Wei et al. proposed a double-layer collaborative apportionment method, named $2 \mathrm{~L}-\mathrm{CoV}$, for personalized and balanced routing in transportation system, which can guide traffic flow speed, and plan the routing with considering the driving preferences under the assistance of space-airground integrated networks.

In the article "An ACO-based Cross-layer Routing Algorithm in Space-Air-Ground Integrated Networks", Zheng et al. proposed a novel ACO-based cross-layer routing algorithm for Space-air-ground integrated networks, which takes the link quality and end-to-end packed delay in the physical layer as driving factors to search for the optimal routing path.

In the article "Trust based Task Offloading Scheme in UAV-enhanced Edge Computing Network", Ouyang et al. proposed a trust based intelligent task offloading scheme in the UAV-enhanced edge network, which can effectively reduce both the task loss rate of the network and the total energy consumption of the IoT devices.
In the article "Resource Management of GEO Relays for Real-Time Remote Sensing", Xu et al. proposed a real-time resource management approach for GEO relaying, aiming at maximizing the network throughput and reducing the transmission delay.

In the article "An efficient three-factor remote user authentication protocol based on BPV-FourQ for Internet of Drones", Zhang et al. proposed a lightweight multi-factor authentication and key agreement (AKA) protocol for Internet of Drones (IoD), which can realize the real multi-factor security using smart card, biometric and password, and achieve perfect forward secrecy and anonymity of users.

We would like to express our sincere gratitude to all the authors for submitting their papers and to the reviewers for their valuable comments and suggestions that significantly enhanced the quality of these articles. We are also grateful to Prof. Shen, the Editor-in-Chief of Peer-toPeer Networking and Applications, for his great support throughout the whole review and publication process of this special issue, and, of course, to all the editorial staff for their great helps. We hope that this special issue could be a useful reference for researchers, scientists, engineers, and academics in the field of IoT networks.

Publisher's note Springer Nature remains neutral with regard to jurisdictional claims in published maps and institutional affiliations.

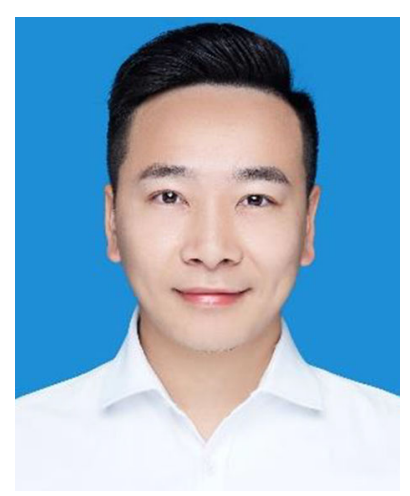

Feng Lyu received the $\mathrm{PhD}$ degree from the Department of Computer Science and Engineering, Shanghai Jiao Tong University, Shanghai, China, in 2018, and the BS degree in software engineering from Central South University, Changsha, China, in 2013. During respective Sept. 2018-Dec. 2019 and Oct. 2016Oct. 2017, he worked as a postdoctoral fellow and was a visiting $\mathrm{PhD}$ student in BBCR Group, Department of Electrical and Computer Engineering, University of Waterloo, Canada. He is currently a professor with the School of Computer Science and Engineering, Central South University, Changsha, China. His research interests include vehicular networks, beyond $5 \mathrm{G}$ networks, big data measurement and application design, and could/edge computing. He is the recipient of the best paper award of IEEE ICC 2019, and Norbert Wiener Review Award 2020 of IEEE/CAA Journal of Automatica Sinica. He currently serves as associate editor for IEEE Systems Journal, leading guest editor for Peer-to-Peer Networking and Applications, and guest editor for China Communications, and served as TPC members for many international conferences. He is a member of the IEEE Computer Society, Communication Society, and Vehicular Technology Society. 


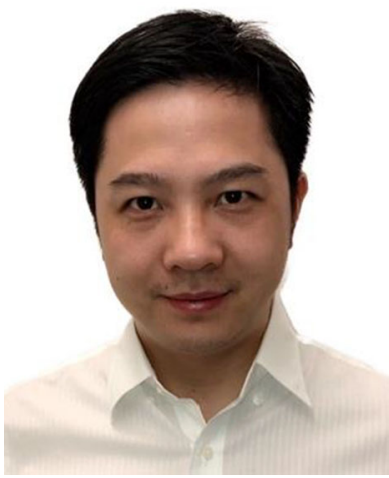

Wenchao $\mathrm{Xu}$ is a research assistant professor at The Hong Kong Polytechnic University. He received his Ph.D. degree from University of Waterloo, Canada, in 2018. Before that he received the B.E. and M.E. degrees from Zhejiang University, Hangzhou, China, in 2008 and 2011, respectively. In 2011, he joined Alcatel Lucent Shanghai Bell Co. Ltd., where he was a Software Engineer for telecom virtualization. He has also been an Assistant Professorat School of Computing and Information Sciences in Caritas Institute of Higher Education, Hong Kong. His research interests include wireless communication, Internet of things, distributed computing and $\mathrm{AI}$ enabled networking.

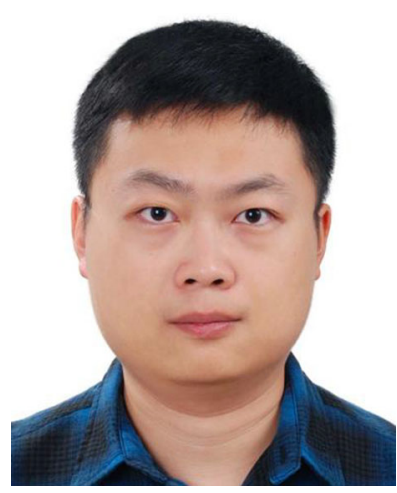

Quan Yuan received his Ph.D. degree in computer science and technology from Beijing University of Posts and Telecommunications, China, in 2018. He is currently a lecturer at the State Key Laboratory of Networking and Switching Technology, BUPT. His current research interest includes mobile computing, crowdsensing, and vehicular networks.

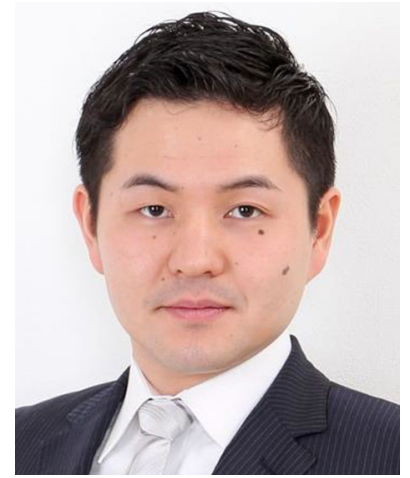

Katsuya Suto received the B.Sc degree in computer engineering from Iwate University, Morioka, Japan, in 2011, and the M.Sc. and Ph.D. degrees in information science from Tohoku University, Sendai, Japan, in 2013 and 2016, respectively. He has worked as a Postdoctoral Fellow for Research Abroad, Japan Society for the Promotionof Science, in the Broadband Communications Research Lab., University of Waterloo, ON, Canada, from 2016 to 2018 . He is currently an Assistant Professor with the Graduate School of Informatics and Engineering, the University of Electro-Communications, Tokyo, Japan. His research interests include mobile edge computing, cognitive radio, green wireless networking, and deep learning. He received the Best Paper Award at the IEEE VTC2013-spring, the IEEE/CIC ICCC2015, the IEEE ICC2016, and the IEEE Transactions on Computers in 2018. He served as Guest Editor for the Peer-to-Peer Networking and Applications in 2020, the IEEE Transactions on Cognitive Communications and Networking in 2019, and the International Journal of Distributed Sensor Networks in 2017. He is currently Associate Editor of the International Journal of Computers and Applications. 